\title{
Vivência e expectativas do cuidador principal de criança obesa
}

\author{
Experience and expectations of the primary caregiver of obese children
}

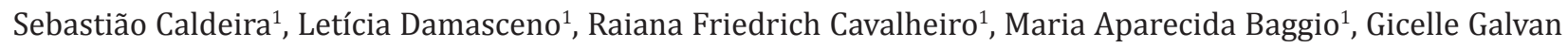
Machineski ${ }^{1}$, Thaissy Fernanda de Oliveira ${ }^{1}$

Objetivo: compreender a vivência e expectativas do cuidador principal de criança obesa. Métodos: pesquisa qualitativa fundamentada na Fenomenologia Social de Alfred Schütz com 16 cuidadores de crianças obesas por meio de entrevistas. Resultados: identificaram-se duas categorias referentes aos "motivos por que": Conhecimento sobre a obesidade infantil e Vivência com a obesidade do(a) filho(a). Quanto aos "motivos para", constataram-se três categorias: 0 que espera para a criança obesa; O que espera para si mesmo; e Expectativas perante os serviços de saúde e escolar. Conclusão: evidenciou-se que o grupo de cuidadores estudados possui conhecimentos sobre o fenômeno obesidade e suas consequências, como também a respeito da inadequação da alimentação e peso dos filhos, porém, ao vivenciá-la, não aceita a condição de obesidade dos seus filhos.

Descritores: Saúde da Criança; Obesidade; Transtornos da Nutrição Infantil; Cuidadores; Pesquisa Qualitativa.

Objective: to understand the experience and expectations of the primary caregiver of obese children. Methods: a qualitative research based on the Social Phenomenology of Alfred Schütz with 16 caregivers of obese children through interviews. Results: two categories were identified regarding the "reasons why": Knowledge about childhood obesity and Experience with the child's obesity. As for the "reasons for", three categories were found: What is expected for the obese child; What is expected for yourself; and Expectations regarding health and school services. Conclusion: it was evidenced that the group of caregivers studied has knowledge about the phenomenon of obesity and its consequences, as well as about the inadequate diet and body weight of the children. Nevertheless when facing it, they do not accept the condition of obesity of their children.

Descriptors: Child Health; Obesity; Child Nutrition Disorders; Caregivers; Qualitative Research.

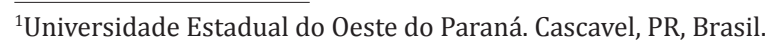




\section{Introdução}

A obesidade e o sobrepeso ocorrem devido ao acúmulo anormal de gordura corporal. Exercem efeitos metabólicos adversos na pressão arterial e nas concentrações de colesterol e triglicérides, contribuindo para o aumento de doenças cardiovasculares, acidentes cerebrovasculares isquêmicos, diabetes mellitus de tipo dois ou alguns tipos de câncer(1). É um problema de saúde pública, uma doença evitável, crônica, provocada pela associação de múltiplos fatores, como comportamento, ambiente, genética, atingindo a população no âmbito mundial, com índices que apontam elevação treplicada, desde 1975. Em 2016, cerca de 1,9 bilhões de adultos com 18 anos ou mais tinham excesso de peso, destes 650 milhões eram obesos ${ }^{(1-2)}$.

No âmbito mundial, as crianças também fazem parte desse grupo de população obesa e com excesso de peso, chegando a 40 milhões em 2010, segundo dados da Organização Mundial de Saúde. Em 2025, caso não sejam tomadas providências adequadas, o número de crianças obesas poderá atingir 75 milhões. No Brasil, 50,0\% da população encontra-se obesa, visto que $21,7 \%$ da população masculina entre 10 e 19 anos apresenta excesso de peso e 5,7\% encontram-se obesos. A população feminina nessa faixa etária mostra menor índice, $19,4 \%$ de sobrepeso e $4,0 \%$ com obesidade ${ }^{(3-4)}$.

No que tange à obesidade infantil e sua relação com a alimentação, a família possui importante papel e pode acrescentar à criança valores, conhecimentos e hábitos que podem promover a saúde ou a doença no que diz respeito aos saudáveis hábitos alimentares. Sabe-se que o comportamento dos pais e familiares na oferta e consumo de refeições especiais como as guloseimas interfere diretamente no excesso de peso na infância ${ }^{(4)}$. Para que haja mudança no comportamento dos pais ou cuidadores quanto à prática alimentar não saudável, faz-se necessário incentivá-los para as atividades educativas individuais ou em grupo como apoio na construção do conhecimento visando à obtenção de resultados que promovam a alimentação saudável e, consequentemente, melhores resultados no controle da obesidade $\mathrm{e}^{(5-6)}$

Nesse sentido, no ano de 2012, criou-se o Plano Intersetorial de Prevenção e Controle da Obesidade com objetivo de prevenir e controlar a obesidade a partir da intersetorialidade, com ações de promoção à alimentação adequada e saudável e à atividade físi$\mathrm{ca}^{(7)}$. Além disso, fatores como o ambiente doméstico, incluindo a prática inadequada de alimentação saudável e a falta de atividade física, são determinantes para o risco de obesidade. Assim, a família como grupo social pode influenciar os hábitos de seus membros, contribuindo para as formas de nutrição dos mesmos, bem como pode determinar as condições de sobrepeso e obesidade dos familiares ${ }^{(8)}$.

Diante das evidências apresentadas, esta pesquisa parte da seguinte inquietação: Como os cuidadores principais vivenciam e significam a situação de obesidade infantil? Assim, o objetivo deste estudo foi compreender a vivência e expectativas do cuidador principal de criança obesa.

\section{Métodos}

Trata-se de uma pesquisa qualitativa com enfoque na Fenomenologia Social de Alfred Schütz. Esta se preocupa com os fenômenos humanos e envolve a relação e a ação social dos indivíduos ${ }^{(9)}$. A pesquisa foi desenvolvida em dois bairros, um na região sul e outro na região norte, na cidade de Cascavel, Paraná, Brasil. A escolha desses bairros se deu em razão de serem bairros com características socioeconômicas distintas. As famílias do bairro, situado na região norte, têm condição de pobreza; já as famílias residentes no bairro da região sul possuem condições sociais mais favoráveis, assim houve possibilidade de obter riqueza nos relatos dos participantes por residirem em pontos extremos da cidade. A seleção dos participantes ocorreu por meio de levantamento do cadastro no Programa Saúde na Escola, tendo como critérios 
de inclusão: ter idade igual ou maior de 18 anos; ser o cuidador principal da criança obesa independente de ser pai, mãe, familiar ou outro. Foram excluídos os cuidadores principais de crianças obesas que não faziam parte no referido programa, aqueles que cuidam esporadicamente da criança obesa.

Foram feitos contatos por telefone e agendados dia e horário para esclarecimentos sobre a pesquisa e sobre o Termo de Consentimento Livre e Esclarecido. Foram convidados 18 cuidadores, destes 2 não foram encontrados no dia da entrevista, totalizando 16 participantes. Todos eram vinculados à Unidade Básica de Saúde ou Estratégia Saúde da Família, casados, do sexo feminino, mães das crianças obesas, com renda familiar entre um e três salários mínimos, todos dedicando-se ao trabalho no lar e ao cuidado da criança.

A coleta de dados ocorreu no período abril de 2017 a março de 2018, por meio de entrevistas semiestruturadas, audiogravadas, com vistas nos "motivos por que", ou seja, o vivido pelos participantes, bem como nos "motivos para", as expectativas dos participantes perante a criança obesa, si mesmo, os serviços e profissionais, com duração média de 20 minutos cada. Realizaram-se duas entrevistas na modalidade de teste piloto do instrumento. Não houve necessidade de mudança do instrumento, visto que havia relatos com conteúdo suficiente para análise. A obtenção dos relatos se deu no domicílio dos participantes, sem interferência de terceiros.

Foram questões relacionadas aos "Motivos por que": Fale-me sobre sua percepção de obesidade; Qual o seu entendimento sobre a obesidade na infância (olhar voltado para as causas, hábitos de vida, alimentação)? Comente sobre a sua vivência em ter e cuidar de uma criança obesa. Quanto aos "Motivos para", os participantes responderam às seguintes questões: 0 que você espera em relação à condição de obesidade da criança? O que você espera para você enquanto cuidador de criança obesa? O que você espera por parte dos serviços e profissionais da saúde e da escola no que diz respeito às atividades desenvolvidas sobre a obesidade da criança?

As entrevistas foram encerradas quando os relatos se mostraram repetitivos e suficientes para análise das categorias concretas do vivido dos cuidadores principais de crianças obesas de acordo com o referencial adotado. Após as entrevistas, os relatos foram transcritos na íntegra pelos pesquisadores; em seguida, a leitura atentiva e repetitiva a fim de identificar as motivações - "motivos por que" e "motivos para". Assim sendo, os "motivos por que" estão relacionados à vivência passada e presente, explicação posterior ao acontecimento, e os "motivos para" se referem às expectativas $^{(9)}$.

A análise dos relatos foi realizada conforme recomendado por pesquisadores da Fenomenologia Social de Alfred Schütz: 1o passo: leitura atentiva e criteriosa de cada relato na íntegra, procurando, primeiramente, identificar e apreender o sentido global da ação social de cada participante; $2^{\circ}$ passo: releitura de cada depoimento com o objetivo de identificar aspectos comuns que expressam os conteúdos relacionados aos "motivos por que" e aos "motivos para"; $3^{\circ}$ 잉 passo: agrupamento dos aspectos comuns conforme convergência de conteúdos para a composição de categorias concretas; 4ํㅡ passo: análise das categorias concretas para a compreensão da ação social; 5ํㅡ passo: constituição do tipo vivido a partir do conjunto de "motivos por que" e "motivos para" expressos na análise das categorias concretas; $6^{\circ}$ passo: discussão do tipo vivido à luz da Fenomenologia Social de Alfred Schütz e outros referenciais relacionados à temática deste estudo ${ }^{(10)}$.

Faz-se necessário ressaltar que o anonimato dos participantes foi preservado, sendo os mesmos identificados com o Código CP (Cuidador Principal), seguido do número de cada entrevista, sendo CP1 a CP16. 0 estudo atendeu aos preceitos éticos com pesquisas que envolvem seres humanos e foi aprovado pelo Comitê de Ética e Pesquisa da Universidade Estadual do Oeste do Paraná, sob parecer favorável no $1.872 .666 / 2016$ 


\section{Resultados}

Os participantes do estudo foram em sua totalidade mães casadas, do lar, nove cursaram o ensino fundamental e sete o ensino médio. Todos são mães das crianças obesas, com renda familiar entre um e três salários mínimos. Os relatos permitiram a análise em categorias concretas do vivido, que abarcam a experiência vivida no tempo passado e presente - "motivos por que" é a intencionalidade da ação - denominada "motivos para".

A partir da análise dos relatos, no que tange aos "motivos por que", que se relacionam ao contexto vivencial dos cuidadores principais de crianças obesas a partir de sua bagagem de conhecimento, identificaram-se duas categorias, quais sejam: Conhecimento sobre a obesidade infantil e Vivência com a obesidade do(a) filho(a).

Na categoria Conhecimento sobre a obesidade infantil, considera-se a obesidade como o excesso de peso das crianças, uma doença que necessita de atenção e relacionada aos hábitos alimentares e de vida. É um peso fora do limite. Então eu acho que é um problema grave (CP 3). A obesidade é uma doença, porque eles podem começar a engordar pequenininho e podem crescer assim (CP 4). Eu sei que faz muito mal à saúde, se você está obeso, você tem dificuldade de andar, de falar, de respirar (CP 6). Falta de uma alimentação saudável e falta de exercício físico (CP 8).

Os cuidadores acrescentam que as crianças têm uma alimentação e hábitos sedentários inadequados. É o que mostra a categoria Causas da obesidade. Se ela comesse do jeito que era para comer, ela não estaria obesa. Tem mais ansiedade, fazendo com que ela não se alimente bem (CP 1). É a alimentação exagerada, comer fora de hora, não almoçar direito (CP 7). É que criança come muita coisa. A gente como mãe, pode controlar a alimentação, mas a gente tem dó (CP 5).

Na categoria Vivência com a obesidade do(a) filho(a), foi possível compreender que a maioria dos cuidadores se sente responsável pelo excesso de peso da criança e por não ter uma atitude firme em relação à alimentação, demonstrando dificuldade em inserir uma alimentação saudável para a criança, bem como aderir aos hábitos de atividade física. Outros não consideram seu filho obeso, mesmo quando identificado sobrepeso ou obesidade. A gente cuida no limite da gente, só que é ela que não quer esse limite. Ela come em excesso. Na escola tem atividade de esporte uma vez na semana. Em casa eu desligo a TV e faço brincar lá fora (CP 2). Eu me preocupo se ela continuar a crescer assim. Vai sofrer preconceito (CP 9). Mas não é só culpa dela, mas também minha. Eu fico com dó e dou as coisas que ela gosta (CP 10).

Diante da complexidade do tema, destaca-se a categoria Hábitos Alimentares. Nesta, os participantes relatam sobre o processo de amamentação, as refeições que a família e as crianças têm no ambiente domiciliar, como também a alimentação da criança na escola. Salienta-se que somente três crianças foram aleitadas em peito materno até aos dois anos de idade. A maioria teve introdução de alimentos aos seis meses de idade. Os relatos explicitam uma alimentação pobre de nutrientes. É muito difícil ele comer salada. Ele gosta é mais de macarrão quando eu faço. Doce ele come bastante, ele chupa pirulito, bala e chiclete (CP 12). De manhã é pão com margarina ou um ovo frito ou uma linguiça se tiver. No almoço e na janta, come carne, arroz, feijão, ovo frito, linguiça e salada. Ela mamou no peito até dois anos e sete messes (CP 11). A partir dos seis meses, comecei a dar "Danoninho", papinha de bolacha, sopinha de feijão com batatinha. Quando ela chorava eu dava sopinha, "Danoninho" ou bolacha. E assim foi aumentando (CP 14).

Foi evidenciado que os participantes deste estudo possuem um diálogo comprometido em relação à alimentação que o(a) filho(a) tem na escola. Ela compra as coisas no mercado e leva para a escola, diz que o lanche da escola é ruim (CP 13). Aquele lanche da escola acho bem fora do normal para eles que estudam. Não tem vitamina nenhuma. Tem vezes que é aquela bolacha doce com suco ou leite achocolatado e às vezes sagu (CP 18).

Em relação às expectativas, ou seja, os "motivos para", diante do tema em tela, constataram-se três categorias: 0 que espera para a criança obesa; 0 que espera para si mesmo; e Expectativas perante os serviços de saúde e da escola.

Na categoria 0 que espera para a criança obesa, as repostas dos participantes fazem relação à mudan- 
ça na alimentação da criança. Acredita-se que com essa mudança de hábitos alimentares as crianças chegarão à adolescência com o peso adequado. Eu acho que enquanto ele vai crescendo, vai emagrecendo (CP 16). Não me preocupo. Espero que ela emagreça, por que eu era gordinha e emagreci (CP 3). Nós estamos tentando mudar os hábitos, comprar mais frutas, essas coisas. Fazer ela se exercitar mais (CP 17).

$\mathrm{Na}$ categoria $\mathrm{O}$ que espera para si mesmo, os participantes esperam que seus filhos não sejam mais obesos e com a mudança nos hábitos alimentares consigam perder peso, minimizar o sentimento de impotência perante a situação vivenciada e formas de enfrentamento. Poucos relataram sobre a necessidade de mudança do hábito sedentário das crianças. Eu espero que no futuro ela não seja obesa, por que na minha família e na do meu marido não tem ninguém obeso (CP 3). Tenho o sentimento de impotência, espero conseguir manter o foco e cuidar da alimentação da minha filha (CP 15). Espero poder cuidar melhor da alimentação do meu filho (CP 8).

$\mathrm{Na}$ categoria Expectativas perante os serviços de saúde e da escola, os participantes esperam que os serviços de saúde atendam de forma resolutiva os seus filhos e que a escola consiga controlar a quantidade de alimentos ofertando alimentação saudável. Espero que eles (escola e posto de saúde) mandem uma lista sobre o que ela pode comer ou não para ela poder emagrecer (CP 4). Espero que a nutricionista passe um jeito melhor de fazer as coisas, para ela conseguir comer (CP 15). Espero que escola ajude, acho que eles sempre deveriam dar alimentação saudável e não deixar as crianças repetirem (CP 6).

\section{Discussão}

Este estudo tem limitações por lançar o olhar para o fenômeno obesidade somente aos cuidadores. É preciso ampliar esse horizonte com novas pesquisas sobre o olhar das crianças e dos adolescentes, bem como o olhar dos profissionais da saúde e da educação. Ressalta-se que esses segmentos estão sendo estudados em outros recortes de pesquisas vinculadas ao projeto maior que originou o presente estudo.

Os resultados obtidos neste trabalho contri- buem para a reflexão sobre o cotidiano vivenciado pelos cuidadores principais de crianças obesas ao se depararem com suas próprias angústias e limitações relacionadas à obesidade e entorno, como o conhecimento incipiente sobre agravantes da obesidade infantil, aos hábitos de vida e à alimentação. Por ser um fenômeno persistente e ao mesmo tempo emergente, complexo e desafiador para toda a sociedade, a obesidade infantil merece destaque na pauta da saúde pública visando resolubilidade do problema, não ficando apenas no âmbito da reflexão sobre a temática.

No âmbito do ensino e da pesquisa, espera-se que os resultados aqui encontrados possam suscitar outros estudos sobre a obesidade infantil. No que diz respeito à assistência e à vivência dos cuidadores, que este estudo possa sensibilizar e instigar o cuidado mais eficaz por parte dos órgãos responsáveis e que os profissionais da saúde e da educação e os cuidadores principais encontrem estratégias de cuidado que sejam resolutivas para a criança obesa.

É necessário sensibilizar tanto os profissionais quanto os familiares em relação à inadequação da alimentação e do peso do(a) filho(a) e das consequências da obesidade desmistificando a ideia de que o excesso de peso se resolve com o crescimento e desenvolvimento da criança. Esse entendimento se torna possível por meio das experiências e vivências, bem como da bagagem de conhecimentos adquiridos de antecessores, de pessoas do convívio, como os familiares vivenciando a obesidade de um filho, ou por meio de conhecimentos escolares, estudos, leituras entre outros ${ }^{(9)}$.

Identifica-se que o desenvolvimento de sobrepeso e obesidade infantil não estão relacionados somente aos aspectos socioeconômicos, como revela outro estudo ${ }^{(11)}$. Logo, os cuidadores revelam má percepção do estado nutricional dos filhos pelos familiares, que tendem a subestimar a obesidade por não possuir efeito nocivo imediato na saúde da criança, na maioria dos $\operatorname{casos}^{(12)}$.

Embora os cuidadores reconhecem a obesidade como doença, nota-se que não reconhecem a situação 
de obesidade da criança, pois acreditam que enquanto cresce, emagrece. Assim, confirma-se que o sobrepeso e a obesidade infantil são carregados de estigma, o que se coloca como uma barreira para a discussão do peso da criança com a família. Compete aos profissionais da saúde a tarefa de levantar a discussão sobre a temática de modo a orientar e sensibilizar os cuidadores $^{(13)}$.

Nesse contexto, os profissionais de saúde podem ajudá-los a aceitar o sobrepeso infantil e reconhecer necessidade de mudança do comportamento familiar em relação à alimentação. Soma-se a isso a necessidade de intervenções sociais por meio do fortalecimento de políticas públicas e melhor utilização dos serviços de atenção primária, incluindo apoio psicológico $^{(14)}$.

É preciso motivação para chegar ao ideal almejado, a qual se caracteriza pelo "motivos por que", ou seja, toda a bagagem de conhecimentos adquiridos até então sobre a obesidade e sua importância, bem como os "motivos para", o projetar-se para o futuro no que tange ao cuidar-se para diminuir a obesidade e suas consequências à saúde $\mathrm{e}^{(9-10)}$.

Os cuidadores apontam expectativas quanto aos serviços de saúde e escola para melhoria de aspectos nutricionais da alimentação da criança, em qualidade e quantidade. No entanto, salienta-se que são os pais os principais formadores de hábitos alimentares saudáveis na primeira infância, mediadores para as escolhas salutares pelas crianças ${ }^{(15)}$.

Ainda assim, a escola é considerada espaço propício para o desenvolvimento de intervenções e estímulo ao autocuidado. As práticas educativas relacionadas às atividades físicas e aos aspectos alimentares devem ser incentivadas. Sobretudo, a realização de atividade física torna-se imprescindível no combate à obesidade quando sua prática torna-se prazerosa para a criança $\mathrm{a}^{(16-17)}$.

Ações com a escola são o alicerce para uma formação saudável, contudo práticas intersetoriais possuem mais força para atuar na obesidade infantil, como mostra estudo em um município da Grande São
Paulo, com gestores e profissionais da saúde e da educação, o qual revela a implantação de um grupo de trabalho intersetorial que possibilitou um plano de ação pautado na realidade local e nas especificidades das unidades básicas de saúde e das escolas, planejando ações em conjunto com o Programa Saúde na Escola, o Núcleo de Atenção à Saúde da Famíliaa ${ }^{(18)}$.

É imprescindível que os profissionais de saúde fiquem atentos às organizações sociais e seus hábitos e, em conjunto, busquem qualificar a assistência, a qual deve ser prestada de forma completa e individualizada. Assim sendo, faz-se necessário investir em formação permanente dos profissionais das diversas áreas que atuam nas atividades de prevenção e promoção à saúde da criança para que a obesidade seja vista e pensada por equipe multiprofissional ou interdisciplinar, chegando-se, assim, ao esperado e preconizado ${ }^{(18-19)}$.

É perceptível que para reverter o crescente número de casos de obesidade infantil, quer sejam no âmbito nacional, estadual, regional ou local, faz-se necessário adotar ações de saúde por parte da equipe interdisciplinar e em âmbito intersetorial, envolvendo escola e comunidade, visto que todo o estoque de conhecimentos adquirido, experiências e vivências perfazem o que somos hoje, ou seja, a situação biográfica de cada um ${ }^{(9)}$.

Para que a obesidade possa ser revertida ainda na infância, é preciso renovar o estoque de conhecimentos sobre a mesma, permitindo, desse modo, situar-se biograficamente de forma diferente. Isso consequentemente estará envolto de mudanças nos hábitos alimentares e de vida. Ressalta-se que crianças e adolescentes com sobrepeso ou obesa tendem a manter essa condição nutricional em todas as fases da vida, o que torna elevada essa prevalência de alterações nutricionais no adulto e no idoso(20).

No âmbito dos serviços, cabe aos profissionais de saúde, em particular da equipe de Enfermagem, renovar sua bagagem de conhecimentos quanto à educação em saúde voltada para a família e comunidade, bem como as ações voltadas ao planejamento 
e construção de estratégias para o acompanhamento da obesidade em sua área de abrangência. Para tanto, faz-se necessário atuar de forma objetiva e subjetiva. É preciso chegar-se à subjetividade das pessoas sob o cuidado profissional, isso pode ser a chave para a adesão ao tratamento e cuidado, visando adquirir novos estoques de conhecimentos e, principalmente, ter a mudança de hábitos e estilos de vida no que tange ao processo saúde e doença no contexto da obesidade.

\section{Conclusão}

Evidenciou-se que o grupo de cuidadores estudados possui conhecimentos sobre o fenômeno obesidade e suas consequências, como também a respeito da inadequação da alimentação e peso dos filhos, porém, ao vivenciá-la, não aceita a condição de obesidade dos seus filhos.

\section{Agradecimentos}

À Fundação Araucária pelo financiamento da pesquisa por meio do Edital no 09/2016.

\section{Colaborações}

Caldeira S, Damasceno L, Cavalheiro RF, Baggio MA e Oliveira TF contribuíram na análise e interpretação dos dados, na redação do artigo e revisão crítica relevante do conteúdo intelectual. Machineski GG contribuiu na concepção do projeto. Todos os autores contribuíram na aprovação final da versão a ser publicada.

\section{Referências}

1. Russell CG, Taki S, Laws R, Azadi L, Campbell KJ, Elliott R, et al. Effects of parent and child behaviors on overweight and obesity in infants and young children from disadvantaged backgrounds: systematic review with narrative synthesis. BMC Public Health. 2016; 16:151. doi: https://doi. org/10.1186/s12889-016-2801-y
2. Salvador CCZ, Kitoko PM, G AMD. Nutritional status of children and adolescents: factors associated to overweight and fat accumulation. J Hum Growth. 2014; 24(3):313-9. doi: http://dx.doi. org/10.7322/jhdg.88969

3. Marques MS, Silva JR, Lima CAG, Maia EMGC. Prevalência de sobrepeso e obesidade entre crianças de 7 a 10 anos atendidas em unidade de Estratégia Saúde da Família - ESF. Rev Bras Med Fam Comunidade. 2015; 10(37):1-9. doi: http://rbmfc.org. br/rbmfc/article/view/1032/743

4. Michereff ALP, Tachini L, Mezadri T, Hofelman DA. Associação da qualidade da dieta com as características do ambiente familiar em escolares de Itajaí-SC. Mundo Saúde [Internet]. 2014 [citado 2019 Mai 22];38(3):296-305. Disponível em: http://www.saocamilo-sp.br/pdf/mundo_saude/155565/A06.pdf

5. Melo KM, Cruz ACP, Brito MFSF, Pinho L. Influence of parents' behavior during the meal and on overweight in childhood. Esc Anna Nery. 2017; 21(4):1-6. doi: http://dx.doi.org/10.1590/21779465-ean-2017-0102

6. Moura IH, Silva AN, Anjos JS, Castro THU, Almeida PC, Silva ARV. Educational strategies with adolescents at risk from diabetes type 2: comparative study. Online Braz J Nurs [Internet]. 2015 [cited Mai 18, 2019]; 14(1):25-31. Available from: http://www.objnursing.uff.br/index.php/nursing/article/view/4585

7. Paiva LKF, Cunha Júnior AT, Knackfuss MI, Medeiros HF. Obesidade em adolescentes e as políticas públicas de nutrição. Cienc Saúde Coletiva. 2014; 19(6):1755-62. doi: http://dx.doi. org/10.1590/1413-81232014196.12382013

8. Duré ML, Schwanke NL, Borges TS, Burgos MS, Garcia EL, Krug SF. A obesidade infantil: um olhar sobre o contexto familiar, escolar e da mídia. $\mathrm{R}$ Epidemiol Control Infec. 2015; 5(4):191-6. doi: http://dx.doi.org/10.17058/reci.v5i4.6072

9. Schütz A. Sobre fenomenologia e relações sociais. Petrópolis: Vozes; 2012.

10. Caldeira S, Luz MS, Tacla MTGM, Machineski GG, Silva RMM, Pinto MPV, et al. Nursing care actions in the paranaense mother network program. Rev Min Enferm. 2017; 21:e-992. doi: http://dx.doi. org/10.5935/1415-2762.20170002 
11. Miranda MQ Palmeira MV, Polito LFT, Brandão MRF, Bocalini DS, Figueira Junior AJ, et al. Prevalência de sobrepeso e obesidade infantil em instituições de ensino: Públicas vs. Privadas. Rev Bras Med Esporte. 2015; 21(2):104-107. doi: http://dx.doi. org/10.1590/1517-869220152102143660

12. Francescatto C, Santos NS, Coutinho VF, Costa RF. Percepção de mães sobre o estado nutricional de seus filhos com excesso de peso: revisão sistemática. J Pediatr. 2014; 90(4):332-43. doi: http://dx. doi.org/10.1590/S0080-623420160000600009

13. Robson SM, Ziegler ML, McCullough MB, Stough CO, Zion C, Simon SL, et al. Changes in diet quality and home food environment in preschool children following weight management. Int $\mathrm{J}$ Behav Nutr Phys Act. 2019; 16:16. doi: https://doi. org/10.1186/s12966-019-0777-6

14. Sutaria S, Saxena S. How can family physicians contribute to ending childhood obesity? Fam Med. 2019; 51(4):308-310. https://doi.org/10.22454/ FamMed.2019.181036

15. Oliveira ND, Silva EB, Ponciano CS, Pontes EDS, Araújo MGG, Oliveira CCA, et al. A importância da formação de hábitos alimentares saudáveis na primeira infância: uma revisão de literatura. Int J Nutr. 2018; 11(1). doi: https://doi.org/10.22454/ FamMed.2019.18103610.1055/s-0038-1674313
16. Ferreira AS, Moraes JRMM, Góes FGB, Silva LF, Broca PV, Duarte SCM. Nursing actions for overweight and obese children in the Family Health Strategy. Rev Rene. 2019; 20:e33892. doi: http://dx.doi. org/10.15253/2175-6783.20192033892

17. Fisberg M, Maximino P, Kain J, Kovalskys I. Obesogenic environment - intervention opportunities. J Pediatr. 2016; 92(3):30-9. doi: http://dx. doi.org/10.1016/j.jped.2016.02.007

18. Batista MSA. Proposta de plano de ação, no âmbito do Programa Saúde na Escola, para prevenção e controle da obesidade infantil em um Município da Grande São Paulo - SP. Bol Inst Saúde [Internet]. 2019; [citado 2019 Ago 13];20(1):52-8. Disponível em: http://docs.bvsalud.org/biblioref/2019/08/1008667/proposta-de-plano_bis_ mestrado_7.pdf

19. Dias PC, Henriques P, Anjos LA, Burland L. Obesity and public policies: the Brazilian government's definitions and strategies. Cad Saúde Pública. 2017; 33(7):e00006016. doi: http://dx.doi. org/10.1590/0102-311x00006016

20. Souza MCC, Tibúrcio JD, Bicalho JMF, Rennó HMS, Dutra JS, Campos LG, et al. Factors associated with obesity and overweight in school-aged children. Texto Contexto Enferm. 2014; 23(3):712-9. doi: dx.doi.org/10.1590/0104-07072014001740013 